\title{
Personalized neural language models for real-world query auto completion
}

\author{
Nicolas Fiorini \\ National Center for Biotechnology Information \\ National Library of Medicine, NIH \\ Bethesda, MD, USA \\ nicolas.fiorini@nih.gov
}

\author{
Zhiyong Lu \\ National Center for Biotechnology Information \\ National Library of Medicine, NIH \\ Bethesda, MD, USA \\ zhiyong.lu@nih.gov
}

\begin{abstract}
Query auto completion (QAC) systems are a standard part of search engines in industry, helping users formulate their query. Such systems update their suggestions after the user types each character, predicting the user's intent using various signals - one of the most common being popularity. Recently, deep learning approaches have been proposed for the QAC task, to specifically address the main limitation of previous popularity-based methods: the inability to predict unseen queries. In this work we improve previous methods based on neural language modeling, with the goal of building an end-to-end system. We particularly focus on using real-world data by integrating user information for personalized suggestions when possible. We also make use of time information and study how to increase diversity in the suggestions while studying the impact on scalability. Our empirical results demonstrate a marked improvement on two separate datasets over previous best methods in both accuracy and scalability, making a step towards neural query auto-completion in production search engines.
\end{abstract}

\section{Introduction}

Predicting the next characters or words following a prefix has had multiple uses from helping handicapped people (Swiffin et al., 1987) to, more recently, helping search engine users (Cai et al., 2016). In practice, most search engines today use query auto completion (QAC) systems, consisting of suggesting queries as users type in the search box (Fiorini et al., 2017). The task suffers from high dimensionality, because the number of possible solutions increases as the length of the target query increases. Historically, the query prediction task has been addressed by relying on query logs, particularly the popularity of past queries (BarYossef and Kraus, 2011; Lu et al., 2009). The idea is to rely on the wisdom of the crowd, as popular queries matching a typed prefix are more likely to be the user's intent.

This traditional approach is usually referred to as MostPopularCompletion (MPC)(Bar-Yossef and Kraus, 2011). However, the performance of MPC is skewed: it is very high for popular queries and very low for rare queries. At the extreme, MPC simply cannot predict a query it has never seen. This becomes a bigger problem in academic search (Lankinen et al., 2016), where systems are typically less used, with a wider range of possible queries. Recent advances in deep learning, particularly in semantic modeling (Mitra and Craswell, 2015) and neural language modeling (Park and Chiba, 2017) showed promising results for predicting rare queries. In this work, we propose to improve the state-of-the-art approaches in neural QAC by integrating personalization and time sensitivity information as well as addressing current MPC limitations by diversifying the suggestions, thus approaching a production-ready architecture.

\section{Related work}

\subsection{Neural query auto completion}

While QAC has been well studied, the field has recently started to shift towards deep learningbased models, which can be categorized into two main classes: semantic models (using Convolutional Neural Nets, or CNNs) (Mitra and Craswell, 2015) and language models (using Recurrent Neural Nets, or RNNs) (Park and Chiba, 2017). Both approaches are frequently used in natural language processing in general (Kim et al., 2016) and tend to capture different features. In this work, we focus on RNNs as they provide a flexible solution to generate text, even when it is not previously seen in the training data.

Yet, recent work in this field (Park and Chiba, 2017) suffers from some limitations. Most importantly, the probability estimates for full queries 
are directly correlated to the length of the suggestions, consequently favoring shorter queries in some cases and hampering some predictions (Park and Chiba, 2017). By appending these results to MPC's and re-ranking the list with LambdaMART (Burges, 2010) in another step as suggested in previous work (Mitra and Craswell, 2015), they achieve state-of-the-art performance in neural query auto completion at the cost of a higher complexity and more computation time.

\subsection{Context information}

Still, these preliminary approaches have yet to integrate standards in QAC, e.g. query personalization (Koutrika and Ioannidis, 2005; Margaris et al., 2018) and time sensitivity (Cai et al., 2014). This integration has to differ from traditional approaches by taking full advantage of neural language modeling. For example, neural language models could be refined to capture interests of some users as well as their actual language or query formulation. The same can apply to timesensitivity, where the probability of queries might change over time (e.g. for queries such as "tv guide", or "weather"). Furthermore, the feasibility of these approaches in real-world settings has not been demonstrated, even more so on specialized domains.

By addressing these issues, we make the following contributions in this work compared to the previous approaches:

- We propose a more straightforward architecture with improved scalability;

- Our method integrates user information when available as well as time-sensitivity;

- We propose to use a balanced beam search for ensuring diversity;

- We test on a second dataset and compare the generalizability of different methods in a specialized domain;

- Our method achieves stronger performance than the state of the art on both datasets.

Finally, our source code is made available in a public repository ${ }^{1}$. This allows complete reproducibility of our results and future comparisons.

\footnotetext{
${ }^{1}$ https://github.com/ncbi-nlp/NQAC
}

\section{Methods}

\subsection{Personalized neural Language Model}

The justification of using a neural language model for the task of predicting queries is that it has been proven to perform well to generate text that has never been seen in the training data (Sutskever et al., 2011). Particularly, character-level models work with a finer granularity. That is, if a given prefix has not been seen in the training data (e.g. a novel or incomplete word), the model can use the information shared across similar prefixes to make a prediction nonetheless.

Recurrent Neural Network The difficulty of predicting queries given a prefix is that the number of candidates explodes as the query becomes longer. RNNs allow to represent each character (or word) of a sequence as a cell state, therefore reducing the dimensionality of the task. However, they also introduce the vanishing gradient problem during backpropagation, preventing them from learning long-term dependencies. Both gated recurrent units (GRU) (Cho et al., 2014) and long-short term memory cells (LSTMs) solve this limitation - albeit with a different approach and are increasingly used. In preliminary experiments, we tried various forms of RNNs: vanilla RNNs, GRUs and LSTMs. GRUs performed similarly to LSTM with a smaller computational complexity due to fewer parameters to learn as was previously observed (Jozefowicz et al., 2015).

\section{Word embedded character-level Neural} Language Model The main novelty in (Park and Chiba, 2017) is to combine a character-level neural language model with a word-embedded space character. The incentive is that character-level neural language models benefit from a finer granularity for predictions but they lack the semantic understanding words-level models provide, and vice versa. Therefore, they encode text sequences using one-hot encoding of characters, character embedding and pre-trained word embedding (using word2vec (Mikolov et al., 2013)) of the previous word when a space character is encountered. Our preliminary results showed that the character embedding does not bring much to the learning, so we traded it with the context feature vectors below to save some computation time while enriching the model with additional, diverse information. 
User representation We make the assumption that the way a user types a query is a function of their actual language/vocabulary, but also a function of their interests. Therefore, a language model could capture these user characteristics to better predict the query, if we feed the learner with the information. Each query $q_{i}$ is a set of words such that $q_{i}=\left\{w_{1}, \ldots, w_{n}\right\} . U$ is a column matrix and a user $u \in U$ is characterized by the union of words in their $k$ past queries, i.e. $Q^{u}=\cup_{i=1}^{k} q_{i}$. The objective is to reduce, for each user, the vocabulary used in their queries to a vector of a dimensionality $d$ of choice, or $Q^{u} \rightarrow \mathbb{R}^{d}$. We chose $d=30$, in order to stay in the same computation order of previous work using character embedding (Park and Chiba, 2017). To this end, we adapted the approach $P V-D B O W$ detailed in (Le and Mikolov, 2014). That is, at each training iteration, a random word $w_{i}$ is sampled from $Q^{u}$. The model is trained by maximizing the probability of predicting the user $u$ given the word $w_{i}$, i.e.:

$$
\frac{1}{|U|} \sum_{u \in U} \sum_{w_{i} \in Q^{u}} \log P\left(u \mid w_{i}\right) .
$$

The resulting vectors are stored for each user ID and are used as input for the neural net (NN) (see Architecture section).

Time representation As an example, in the background data (see Section 4.1), the query "tv guide" appears 1,682 times and it is vastly represented in evening and nights. For this reason, we propose to integrate time features in the language model. While there has been more elaborated approaches to model it in the past (Shokouhi and Radinsky, 2012), we instead propose a straightforward encoding and leave the rest of the work to the neural net. For each query, we look at the time it was issued, consisting of hour $x$, minute $y$ and second $z$, and we derive the following features:

$$
\begin{aligned}
& \sin \left(\frac{2 \pi(3600 x+60 y+z)}{86400}\right), \\
& \cos \left(\frac{2 \pi(3600 x+60 y+z)}{86400}\right) .
\end{aligned}
$$

This encoding has the benefit of belonging to $[-1,1]$, which is a range comparable to the rest of the features. It is also capable to model cyclic data, which is important particularly around boundaries (e.g. considering a query at 11:55PM and another at 00:05AM). We proceed the same way to encode weekdays and we end up with four time features.

Overall architecture An overview of the architecture is proposed in Figure 1. The input of our neural language model is a concatenation of the vectors defined above, for each character and for each query in the training set. We use zeropadding after the " $\backslash \mathrm{n}$ " character to keep the sequence length consistent, and the NN learns to recognize it. We feed this input vector into 2 layers of $1024 \mathrm{GRUs}^{2}$, each followed by a dropout layer (with a dropout rate of 50\%) to prevent overfitting. Each GRU cell is activated with $\operatorname{Re} L u(x)=x^{+}$ and gradients are clipped to a norm of 0.5 to avoid gradient exploding problems. The output of the second dropout layer is fed to a temporal softmax layer, which allows to make predictions at each state. The softmax function returns the probability $P\left(c_{i} \mid c_{1}, \ldots, c_{i-1}\right)$ of the character $c_{i}$ given the previous characters of the sequence, which is then used to calculate the loss function by comparing it to the next character in the target query. Instead of using the objective denoted in (Park and Chiba, 2017), we minimize the loss $\mathcal{L}$ defined as the average cross entropy of this probability with the reference probability $\hat{P}\left(c_{i}\right)$ across all queries, that is

$$
\begin{gathered}
\mathcal{L}= \\
-\frac{1}{|Q|} \sum_{q \in Q} \sum_{i=1}^{|q|-1} \hat{P}\left(c_{i+1}\right) \times \log P\left(c_{i+1} \mid c_{1}, \ldots, c_{i}\right) .
\end{gathered}
$$

$Q$ is the set of queries in the training dataset, $|Q|$ is the total number of queries in the set and $|q|$ is the number of characters in the query $q$. Convergence stabilizes around 5-10 epochs for the AOL dataset (depending on the model) and 15-20 epochs for the biomedical specialized dataset (see Section 4.1).

\subsection{Balanced diverse beam search}

The straightforward approach for decoding the most likely output sequence - in this case, a suffix given a prefix - is to use a greedy approach. That is, we feed the prefix into the trained NN and pick the most likely output at every step, until the sequence is complete. This approach has a high

\footnotetext{
${ }^{2}$ It was reported that using more cells may not help the prediction while hurting computation (Park and Chiba, 2017).
} 


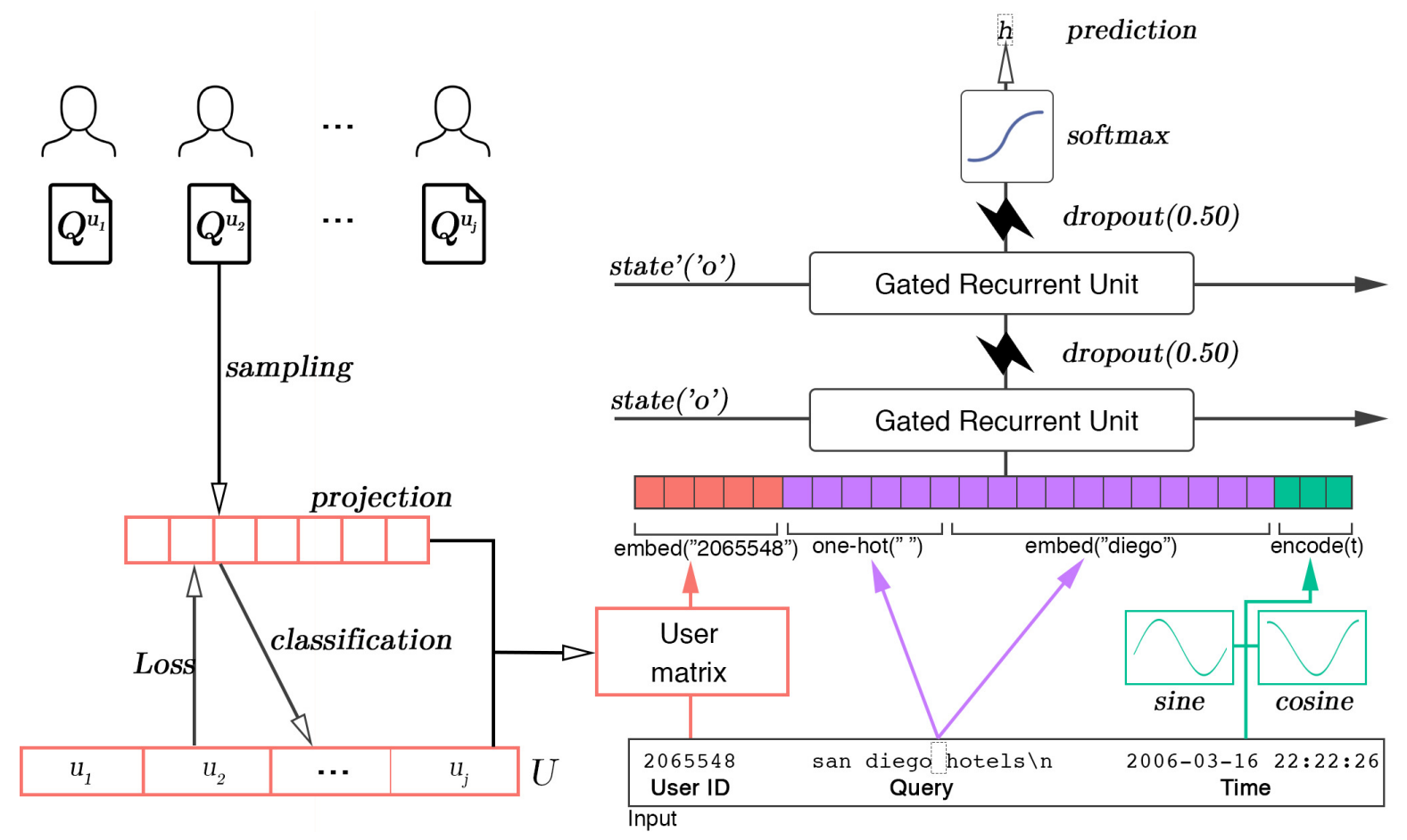

Figure 1: Architecture of our proposed model.

chance to output a locally optimal sequence and a common alternative is to use a beam search instead. We propose to improve the beam search by adding a greedy heuristic within it, in order to account for the diversity in the results. A similar suggestion has been made in (Vijayakumar et al., 2016), and our proposition differs by rebalancing the probabilities after diversity was introduced. In (Vijayakumar et al., 2016), at every step the most likely prediction is not weighted while all others are, by greedily comparing them. This approach effectively always prefers the most likely character over all other alternatives at each step. The first result will thus be the same as the local optimum using a greedy approach, which becomes problematic for QAC where order is critical. By rebalancing the probability of the most likely suggestion with the average diversity weight given to other suggestions, we make sure probabilities stay uniform yet suggestions are diverse. We use a normalized Levenshtein distance to assess the diversity.

\section{Experiments}

\subsection{Dataset}

The AOL query logs (Pass et al., 2006) are commonly used to evaluate the quality of QAC systems. We rely on a background dataset for the
$\mathrm{NN}$; training and validation datasets for lambdaMART integrations; and a test dataset for evaluations. Some adaptations are done to the AOL background dataset as in (Park and Chiba, 2017), such as removing the queries appearing less than 3 times or longer that 100 characters. For each query in the training, validation and test datasets, we use all possible prefixes starting after the first word as in (Shokouhi, 2013). We use the sets from (Park and Chiba, 2017) available online, enriched with user and time information provided in the original AOL dataset. In addition, we evaluate the systems on a second real-world dataset from a production search engine in the biomedical domain, PubMed (Fiorini et al., 2017; Lu, 2011; Mohan et al., 2018), that was created in the same manner. The biomedical dataset consists of $8,490,317$ queries. The sizes of training, validation and test sets are comparable to those used for the AOL dataset.

\subsection{Evaluation}

Systems are evaluated using the traditional Mean Reciprocal Rank (MRR) metric. This metric assesses the quality of suggestions by identifying the rank of the real query in the suggestions given one of its prefixes. We also tested PMRR as introduced in (Park and Chiba, 2017) and observed the same trends in results as MRR, so we do not show them due to space limitation. Given the set of prefixes 
$P$ in the test dataset, MRR is defined as follows:

$$
M R R=\frac{1}{|Q|} \sum_{r \in P} \frac{1}{r_{p}}
$$

where $r_{p}$ represent the rank of the match. Paired t-tests measure the significance of score variations among systems and are reported in the Results section. We also evaluate prediction time as this is an important parameter for building production systems. The prediction time is averaged over 10 runs on the test set, on the same hardware for all models. We do not evaluate throughput but rather compare the time required by all approaches to process one prefix.

\subsection{Systems and setups}

We implemented the method in (Park and Chiba, 2017) and used their best-performing model as a baseline. We also compare our results to the standard MPC (Bar-Yossef and Kraus, 2011). For our method, we evaluate several incremental versions, starting with NQAC which follows the architecture detailed above but with the word embeddings and the one-hot encoding of characters only. We add the subscript $\mathbf{U}$ when the language model is enriched with user vectors and $\mathbf{T}$ when it integrates time features. We append $+\mathbf{D}$ to indicate the use of the diverse beam search to predict queries instead of a standard beam search. Finally, we also study the impact of adding MPC and LambdaMART (+MPC, + $\lambda$ MART).

\section{Results}

A summary of the results is presented in Table 1 . Interestingly, our simple NQAC model performs similarly to the state-of-the-art on this dataset, called Neural Query Language Model (NQLM), on all queries. It is significantly less good for seen queries $(-5.6 \%)$ and significantly better for unseen queries $(+4.2 \%)$. Although GRUs have less expressive power than LSTMs, their smaller number of parameters to train allowed them to better converge than all LSTM models we tested, including that of (Park and Chiba, 2017). NQAC also benefits from a significantly better scalability (28\% faster than NQLM) and thus seems more appropriate for production systems. When we enrich the language model with user information, it becomes better for seen queries $(+1.9 \%)$ while being about as fast. Adding time sensitivity does not yield significant improvements on this dataset overall, but improves significantly the performance for seen queries $(+1.7 \%)$. Relying on the diverse beam search significantly hurts the processing time (39\% longer) while not providing significantly better performance. Our integration of MPC differs from previous studies. We noticed that for Web search, MPC performs extremely well and is computationally cheap ( 0.24 seconds). On the other hand, all neural QAC systems are better for unseen queries but struggle to stay under a second of processing time. Since identifying if a query has been seen or not is done in constant time, we route the query either to MPC or to $\mathrm{NQAC}_{U T}$ and we note the overall performance as $\mathrm{NQAC}_{U T}+\mathrm{MPC}$. This method provides a significant improvement over NQLM $(+6.7 \%)$ overall while being faster on average. Finally, appending NQAC $_{U T}$ 's results to MPC's and reranking the list with LambdaMART provides the best results on this dataset, but at the expense of greater computational cost $(+60 \%)$.

While NQAC ${ }_{U T}+\mathrm{MPC}$ appears clearly as the best compromise between performance and quality for the AOL dataset, the landscape changes drastically on the biomedical dataset and the quality drops significantly for all systems. This shows the potential difficulties associated with real-world systems, which particularly occur in specialized domains. In this case, the drop in performance is mostly due to the fact that biomedical queries are longer and it becomes more difficult for models to predict the entire query accurately only with the first keywords. While the generated queries make sense and are relevant candidates, the chance for generative models to predict the exact target query diminishes as the target query is longer because of combinatorial explosion. This is even more true when the target queries are diverse as in specialized domains (Islamaj Dogan et al., 2009; Névéol et al., 2011). For example, for the prefix "breast cancer", there are 1169 diverse suffixes in a single day of logs used for training. These include "local recurrence", "nodular prognosis", "hormone receptor", "circulating cells", "family history", "chromosome 4p16" or "herceptin review", to cite only a few. Hence, while the model predicts plausible queries, it is a lot more difficult to predict the one the user intended. The target query length also has an impact on prediction time, as roughly twice the time is needed for Web searches. MPC is the exception, however, it per- 
Table 1: MRR results for all tested models on the AOL and biomedical datasets with their average prediction time in seconds.

\begin{tabular}{|c|c|c|c|c|c|c|c|c|}
\hline \multirow{3}{*}{ Model } & \multicolumn{4}{|c|}{ AOL dataset } & \multicolumn{4}{|c|}{ Biomedical dataset } \\
\hline & \multicolumn{3}{|c|}{ MRR } & \multirow{2}{*}{ Time } & \multicolumn{3}{|c|}{ MRR } & \multirow{2}{*}{ Time } \\
\hline & Seen & Unseen & All & & Seen & Unseen & All & \\
\hline MPC (Bar-Yossef and Kraus, 2011) & 0.461 & 0.000 & 0.184 & 0.24 & 0.165 & 0.000 & 0.046 & 0.29 \\
\hline NQLM(L)+WE+MPC+ $+\lambda$ MART (Park and Chiba, 2017) & 0.430 & 0.306 & 0.356 & 1.33 & 0.159 & 0.152 & 0.154 & 2.35 \\
\hline \multicolumn{9}{|l|}{ Our models in this paper } \\
\hline NQAC & 0.406 & 0.319 & 0.354 & 0.94 & 0.155 & 0.139 & 0.143 & 1.73 \\
\hline $\mathrm{NQAC}_{U}$ & 0.417 & 0.325 & 0.361 & 0.98 & 0.191 & 0.161 & 0.169 & 1.77 \\
\hline $\mathrm{NQAC}_{U T}$ & 0.424 & 0.326 & 0.365 & 0.95 & 0.101 & 0.195 & 0.157 & 1.81 \\
\hline $\mathrm{NQAC}_{U T}+\mathrm{D}$ & 0.427 & 0.326 & 0.366 & 1.32 & 0.186 & 0.185 & 0.185 & 2.04 \\
\hline $\mathrm{NQAC}_{U T}+\mathrm{MPC}$ & 0.461 & 0.326 & 0.380 & 0.68 & 0.165 & 0.195 & 0.187 & 1.20 \\
\hline $\mathrm{NQAC}_{U T}+\mathrm{MPC}+\lambda \mathrm{MART}$ & 0.459 & $\mathbf{0 . 3 3 0}$ & 0.382 & 1.09 & 0.154 & 0.179 & 0.172 & 2.01 \\
\hline
\end{tabular}

forms poorly even on seen queries (0.165). This observation suggests that more elaborate models are specifically needed for specialized domains. On this dataset, NQAC does not perform as well as NQLM and it seems this time that the higher number of parameters in NQLM is more appropriate for the task. Still, user information helps significantly for seen queries $(+23 \%)$, probably because some users frequently check the same queries to keep up-to-date. Time sensitivity seems to help significantly unseen queries $(+21 \%)$ while significantly hurting the quality for seen queries $(-47 \%)$. Diversity is significantly helpful on this dataset $(+19 \%)$ and provides a balance in performance for both seen and unseen queries. $\mathrm{NQAC}_{U T}+\mathrm{MPC}$ yields the best overall MRR score for this dataset, and LambdaMART is unable to learn how to rerank the suggestions, thus decreasing the score.

From these results, we draw several conclusions. First, MPC performs very well on seen queries for Web searches and it should be used on them. For unseen queries, the NQAC $\mathrm{C}_{U T}$ model we propose achieves a sub-second state-of-the-art performance. Second, it is clear that the field of application will affect many of the decisions when designing a QAC system. On a specialized domain, the task is more challenging: fast approaches like MPC perform too poorly while more elaborate approaches do not meet production requirements. $\mathrm{NQAC}_{U}$ performs best on seen queries, NQAC ${ }_{U T}$ on unseen queries. Finally, $\mathrm{NQAC}_{U T}+\mathrm{D}$ provides an equilibrium between the two at a greater computational cost. Its overall MRR is similar to that of NQAC ${ }_{U T}+$ MPC but it is less redundant (see Table 2). Particularly, the system seems not to be limited anymore by the higher probability associ-
Table 2: Comparison of the 10 top query candidates from the baselines and our approach for the prefix

\begin{tabular}{lll} 
"WWW". & & \\
\hline MPC & (Park and Chiba, 2017) & NQAC+D \\
\hline www google com & www google com & www google com \\
www yahoo com & www yahoo com & www myspace com \\
www myspace com & www myspace com & www mapquest com \\
www google & www google & www yahoo com \\
www ebay com & www hotmail com & www hotmail com \\
www hotmail com & www my & www bankofamerica com \\
www mapquest com & www myspace com & www chase com \\
www myspace & www mapquest com & www disneychannel com \\
www msn com & www yahoo & www myspace \\
www bankofamerica com & www disney channel com & www disney channel com \\
\hline
\end{tabular}

ated with shorter suggestions (e.g. "www google", a form of "www google com"), thus bringing more diversity. This aspect can be more useful for specialized domains where the range of possible queries is broader. Finally, we found that a lot more data was needed for the biomedical domain than for general Web search. After about a million queries, NQAC suggests meaningful and plausible queries for both datasets. However, for the biomedical dataset, the loss needs more epochs to stabilize than for the AOL dataset, mainly due to the combinatorial explosion mentioned above.

\section{Conclusions and future work}

To the best of our knowledge, we proposed the first neural language model that integrates user information and time sensitivity for query auto completion with a focus on scalability for real-world systems. Personalization is provided through pretrained user vectors based on their past queries. By incorporating this information and by adapting the architecture, we were able to achieve stateof-the-art performance in neural query auto completion without relying on re-ranking, making this approach significantly more scalable in practice. 
We studied multiple variants, their benefits and drawbacks for various use cases. We also demonstrate the utility of this method for specialized domains such as biomedicine, where the query diversity and vocabulary are broader and MPC fails to provide the same performance as in Web search. We also found that user information and diversity improve the performance significantly more than for Web search engines. To allow readers to easily reproduce, evaluate and improve our models, we provide all the code on a public repository.

The handling of time-sensitivity may benefit from a more elaborate integration, for example sessionbased rather than absolute time. Also, we evaluated our approaches on a general search setup for both datasets, while searches in the biomedical domain commonly contain fields (i.e. authors, title, abstract, etc.) which adds to the difficulty. The choice of a diversity metric is also important and could be faster or more efficient (e.g., using word embeddings to diversify the semantics of the suggestions). These limitations warrant further work and we leave them as perspectives.

\section{Acknowledgement}

This research was supported by the Intramural Research Program of the NIH, National Library of Medicine.

\section{References}

Ziv Bar-Yossef and Naama Kraus. 2011. Contextsensitive query auto-completion. In Proceedings of the 20th international conference on World wide web, pages 107-116. ACM.

Christopher JC Burges. 2010. From ranknet to lambdarank to lambdamart: An overview. Learning, 11(23-581):81.

Fei Cai, Maarten De Rijke, et al. 2016. A survey of query auto completion in information retrieval. Foundations and Trends $\mathrm{R}$ in Information Retrieval, 10(4):273-363.

Fei Cai, Shangsong Liang, and Maarten De Rijke. 2014. Time-sensitive personalized query autocompletion. In Proceedings of the 23rd ACM international conference on conference on information and knowledge management, pages 1599-1608. ACM.

Kyunghyun Cho, Bart Van Merriënboer, Dzmitry Bahdanau, and Yoshua Bengio. 2014. On the properties of neural machine translation: Encoder-decoder approaches. arXiv preprint arXiv:1409.1259.
Nicolas Fiorini, David J Lipman, and Zhiyong Lu. 2017. Cutting edge: Towards pubmed 2.0. eLife, 6:e28801

Rezarta Islamaj Dogan, G Craig Murray, Aurélie Névéol, and Zhiyong Lu. 2009. Understanding pubmed $\mathrm{R}$ user search behavior through log analysis. Database, 2009.

Rafal Jozefowicz, Wojciech Zaremba, and Ilya Sutskever. 2015. An empirical exploration of recurrent network architectures. In International Conference on Machine Learning, pages 2342-2350.

Yoon Kim, Yacine Jernite, David Sontag, and Alexander M Rush. 2016. Character-aware neural language models. In $A A A I$, pages 2741-2749.

Georgia Koutrika and Yannis Ioannidis. 2005. A unified user profile framework for query disambiguation and personalization. In Proceedings of workshop on new technologies for personalized information access, pages 44-53.

Matti Lankinen, Hannes Heikinheimo, Pyry Takala, Tapani Raiko, and Juha Karhunen. 2016. A character-word compositional neural language model for finnish. arXiv preprint arXiv:1612.03266.

Quoc Le and Tomas Mikolov. 2014. Distributed representations of sentences and documents. In International Conference on Machine Learning, pages 1188-1196.

Zhiyong Lu. 2011. Pubmed and beyond: a survey of web tools for searching biomedical literature. Database, 2011.

Zhiyong Lu, W John Wilbur, Johanna R McEntyre, Alexey Iskhakov, and Lee Szilagyi. 2009. Finding query suggestions for pubmed. In AMIA Annual Symposium Proceedings, volume 2009, page 396. American Medical Informatics Association.

Dionisis Margaris, Costas Vassilakis, and Panagiotis Georgiadis. 2018. Query personalization using social network information and collaborative filtering techniques. Future Generation Computer Systems, 78:440-450.

Tomas Mikolov, Ilya Sutskever, Kai Chen, Greg S Corrado, and Jeff Dean. 2013. Distributed representations of words and phrases and their compositionality. In Advances in neural information processing systems, pages 3111-3119.

Bhaskar Mitra and Nick Craswell. 2015. Query autocompletion for rare prefixes. In Proceedings of the 24th ACM international on conference on information and knowledge management, pages 1755-1758. ACM.

Sunil Mohan, Nicolas Fiorini, Sun Kim, and Zhiyong Lu. 2018. A fast deep learning model for textual relevance in biomedical information retrieval. CoRR, abs/1802.10078. 
Aurélie Névéol, Rezarta Islamaj Doğan, and Zhiyong Lu. 2011. Semi-automatic semantic annotation of pubmed queries: a study on quality, efficiency, satisfaction. Journal of biomedical informatics, 44(2):310-318.

Dae Hoon Park and Rikio Chiba. 2017. A neural language model for query auto-completion. In Proceedings of the 40th International ACM SIGIR Conference on Research and Development in Information Retrieval, pages 1189-1192. ACM.

Greg Pass, Abdur Chowdhury, and Cayley Torgeson. 2006. A picture of search. In InfoScale, volume 152 , page 1 .

Milad Shokouhi. 2013. Learning to personalize query auto-completion. In Proceedings of the 36th international ACM SIGIR conference on Research and development in information retrieval, pages 103 112. ACM.

Milad Shokouhi and Kira Radinsky. 2012. Timesensitive query auto-completion. In Proceedings of the 35th international ACM SIGIR conference on Research and development in information retrieval, pages 601-610. ACM.

Ilya Sutskever, James Martens, and Geoffrey E Hinton. 2011. Generating text with recurrent neural networks. In Proceedings of the 28th International Conference on Machine Learning (ICML-11), pages 1017-1024.

Andrew Swiffin, John Arnott, J Adrian Pickering, and Alan Newell. 1987. Adaptive and predictive techniques in a communication prosthesis. Augmentative and Alternative Communication, 3(4):181-191.

Ashwin K Vijayakumar, Michael Cogswell, Ramprasath R Selvaraju, Qing Sun, Stefan Lee, David Crandall, and Dhruv Batra. 2016. Diverse beam search: Decoding diverse solutions from neural sequence models. arXiv preprint arXiv:1610.02424. 